\title{
EDITORIAL
}

\section{Deciphering the "matrix" in pulmonary vascular remodelling}

\author{
P.M. Hassoun
}

V ascular remodelling, a prominent feature of pulmonary arterial hypertension (PAH), is characterised by changes affecting all layers of the pulmonary vessels. The intima becomes infiltrated with fibroblasts and shows prominent fibrosis. The media is the site of smooth muscle hypertrophy and extension of muscle to normally nonmuscularised peripheral arteries as a result of differentiation of pericytes and/or recruitment of smooth muscle cell (SMC) precursors. Similarly, there is increased production of extracellular matrix (ECM), with deposition of collagen and elastin, contributing to the remodelling of the adventitia. The factors that initiate and/or participate in ECM remodelling have been the focus of investigations mostly in animal models of pulmonary hypertension [1]. It has been postulated that matrix remodelling may be preceded by endothelial dysfunction, with alterations in barrier properties leading to the accumulation of a serum factor in the subendothelial space [2]. This putative factor would, in turn, cause activation of a SMC serine elastase [3]. Several lines of evidence obtained from animal experimentation and human studies support the concept that increased elastolytic activity contributes to the pathogenesis of pulmonary hypertension through remodelling of the ECM. Indeed, elastin fragmentation has been observed in the pulmonary arteries of children afflicted with pulmonary hypertension related to congenital heart defects [4]. In addition, while high elastin turnover in conjunction with increased activity of a serine elastase has been observed at least in monocrotaline-induced experimental pulmonary hypertension [5], treatment with elastase inhibitors prevent vascular remodelling and the development of pulmonary hypertension in this and other models of pulmonary hypertension [6].

Other proteolytic enzymes include matrix metalloproteinases (MMP), which are involved in collagen degradation and are important in the regulation of ECM, cellular migration and growth. MMPs are usually present in latent forms and are cleaved by proteases to active forms. MMP 1, 2, 3 and 9 are produced by SMC of the vascular wall. In the vessel wall, MMPs are modulated by counteracting inhibitors (TIMP), and the balance between these two groups of proteinases determines the overall turnover of matrix proteins. The role of MMPs in maintenance of systemic vessel integrity and remodelling has been well established. Similarly, several animal studies have suggested an important role for MMP in ECM and vascular remodelling in experimental pulmonary

CORRESPONDENCE: P.M. Hassoun, Johns Hopkins University, 5501 Hopkins Bayview Circle, Baltimore, MD 21224, USA. Fax: 1 4105502612. E-mail: Phassoun@jhmi.edu hypertension (for example in response to hypoxia), as well as the de-remodelling occurring in the post-hypoxic recovery phase of the syndrome [7]. Increased MMP activity has been demonstrated in animal models of pulmonary hypertension, and MMP inhibition has been shown to either prevent $[8,9]$ or enhance vascular remodelling. For example, inhibition of MMP by TIMP-1 gene transfer or administration of an inhibitor of MMP, such as doxycycline, produces increased muscularisation and adventitial collagen accumulation in rats exposed to hypoxia [10]. Taken together, these experiments clearly suggest a role for MMP in the pathogenesis of pulmonary hypertension despite apparent conflicting results.

In this issue of the European Respiratory Journal, LEPETIT et al. [11] investigate the expression and activity of MMPs and TIMPs in pulmonary vessels and cultured pulmonary artery SMC obtained from patients with idiopathic pulmonary arterial hypertension (IPAH) and control subjects who had undergone lobectomy for localised cancer. Their results indicate an imbalance between MMPs and TIMPs in the IPAH patients that was not present in controls. In particular, the authors found increased TIMP-1 and decreased MMP-3 expression and activity, but also increased active MMP-2 (by gelatin zymography) in IPAH versus control subjects. They conclude that this imbalance in matrix-degrading proteases and their inhibitors may contribute to matrix accumulation and vessel remodelling in IPAH [11].

Several aspects of this study, in which investigators with strong expertise in matrix collagenases have joined forces with experts in the field of pulmonary hypertension, are worth noting. The authors have combined techniques of molecular expression with immunohistochemical localisation and functional analysis (e.g. in situ zymography) to effectively demonstrate an imbalance in MMP/TIMP in human pulmonary vessels, a finding that had long been suspected based on animal models of pulmonary hypertension. Increased expression of MMP-2 and gelatinolytic activities were co-localised along elastic fibres and the inner elastic lamina, which would certainly lend credence to the hypothesis that this enzyme (with both gelatinolytic and elastase activities) plays an active role in ECM remodelling in part through fragmentation of elastin fibres and the elastic lamina. The authors also examined the production of MMPs and TIMPs from SMCs obtained from explants of pulmonary arteries from patients with IPAH and controls and found striking differences, with SMCs from IPAH patients producing significantly more TIMP-1 and active MMP-2 in their conditioned media as compared with SMCs from control patients. 
While highly encouraging and clearly supporting a dynamic remodelling of ECM in IPAH, these results need to be confirmed in larger studies. In particular, the exact nature of the changes and the specific MMPs whose expression is altered in the remodelling process of the pulmonary vasculature need to be better identified and whether such changes are confined to IPAH or akin to other forms of pulmonary hypertension. Once involvement of MMPs and other elastases is established in ECM remodelling in human pulmonary hypertension, other pressing questions will need to be addressed, such as the role of MMP/TIMP imbalance in SMC migration, proliferation and/or apoptosis. Indeed ECM remodelling and elastin fragmentation appear to be complex phenomenas involving several signalling pathways, such as activation of focal adhesion kinase and members of the mitogen activating protein kinase family, with ominous consequences, such as release of smooth muscle mitogens, presumably from proteoglycan storage sites within the ECM [12, 13]. Another consequence of elastase and MMP activation is the production of tenascin-C, an ECM glycoprotein, which co-localises with proliferating SMC in human vascular lesions of pulmonary hypertension [14] and is thought to be responsible for priming of SMC in their response to mitogens (e.g. basic fibroblast and epidermal growth factors) [15]. Inhibition of MMP activity suppresses tenascin-C expression $[3,16]$. In this context, the multiplicity of MMPs and TIMPs and their heterogeneous activities would certainly indicate not only diverging functions from one species to another, but also a complex mechanism of checks and balances for ECM remodelling.

The study by LEPETIT et al. [11] provides a refreshing glimpse of what appears to be a fascinating journey ahead. Such studies are not easy to accomplish considering the scarcity of human tissue available (obtained essentially from lungs of patients with pulmonary hypertension undergoing lung transplantation), the difficulty in isolating and culturing cells of interest (e.g. smooth muscle cells and fibroblasts) and potential cellular phenotypic changes inherent to the in vitro techniques. Finally, the problem of finding adequate control specimens for these experiments is an additional hurdle. However, despite the enormous difficulties, these human studies provide a necessary complement to animal experimentation and are worth all the effort since deciphering the exact regulatory mechanisms of extracellular matrix remodelling and downstream effects may hold one of the essential keys to potential therapeutic targets in pulmonary hypertension.

\section{REFERENCES}

1 Frisdal E, Gest V, Vieillard-Baron A, et al. Gelatinase expression in pulmonary arteries during experimental pulmonary hypertension. Eur Respir J 2001; 18: 838-845.

2 Rabinovitch M. EVE and beyond, retro and prospective insights. Am J Physiol 1999; 277: L5-L12.

3 Cowan KN, Jones PL, Rabinovitch M. Elastase and matrix metalloproteinase inhibitors induce regression, and tenascin- $C$ antisense prevents progression, of vascular disease. J Clin Invest 2000; 105: 21-34.

4 Rabinovitch M, Bothwell T, Hayakawa $\mathrm{BN}$, et al. Pulmonary artery endothelial abnormalities in patients with congenital heart defects and pulmonary hypertension. A correlation of light with scanning electron microscopy and transmission electron microscopy. Lab Invest 1986; 55: 632-653.

5 Maruyama K, Ye CL, Woo M, et al. Chronic hypoxic pulmonary hypertension in rats and increased elastolytic activity. Am J Physiol 1991; 261: H1716-H1726.

6 Ye CL, Rabinovitch M. Inhibition of elastolysis by SC-37698 reduces development and progression of monocrotaline pulmonary hypertension. Am J Physiol 1991; 261 H1255-H1267.

7 Thakker-Varia S, Tozzi CA, Poiani GJ, et al. Expression of matrix-degrading enzymes in pulmonary vascular remodeling in the rat. Am J Physiol 1998; 275: L398-L406.

8 Vieillard-Baron A, Frisdal E, Raffestin B, et al. Inhibition of matrix metalloproteinases by lung TIMP-1 gene transfer limits monocrotaline-induced pulmonary vascular remodeling in rats. Hum Gene Ther 2003; 14: 861-869.

9 Herget J, Novotna J, Bibova J, Povysilova V, Vankova M, Hampl V. Metalloproteinase inhibition by Batimastat attenuates pulmonary hypertension in chronically hypoxic rats. Am J Physiol Lung Cell Mol Physiol 2003; 285: L199-L208.

10 Vieillard-Baron A, Frisdal E, Eddahibi S, et al. Inhibition of matrix metalloproteinases by lung TIMP-1 gene transfer or doxycycline aggravates pulmonary hypertension in rats. Circ Res 2000; 87: 418-425.

11 Lepetit H, Eddahibi S, Fadel E, et al. Smooth muscle cell matrix metalloproteinases in idiopathic pulmonary arterial hypertension. Eur Respir J 2005; 25: 834-842.

12 Mitani Y, Zaidi SH, Dufourcq P, Thompson K, Rabinovitch M. Nitric oxide reduces vascular smooth muscle cell elastase activity through cGMP-mediated suppression of ERK phosphorylation and AML1B nuclear partitioning. FASEB J 2000; 14: 805-814.

13 Thompson K, Rabinovitch M. Exogenous leukocyte and endogenous elastases can mediate mitogenic activity in pulmonary artery smooth muscle cells by release of extracellular-matrix bound basic fibroblast growth factor. J Cell Physiol 1996; 166: 495-505.

14 Jones PL, Cowan KN, Rabinovitch M. Tenascin-C, proliferation and subendothelial fibronectin in progressive pulmonary vascular disease. Am J Pathol 1997; 150: 1349-1360.

15 Jones PL, Rabinovitch M. Tenascin-C is induced with progressive pulmonary vascular disease in rats and is functionally related to increased smooth muscle cell proliferation. Circ Res 1996; 79: 1131-1142.

16 Cowan KN, Jones PL, Rabinovitch M. Regression of hypertrophied rat pulmonary arteries in organ culture is associated with suppression of proteolytic activity, inhibition of tenascin-C, and smooth muscle cell apoptosis. Circ Res 1999; 84: 1223-1233. 